\title{
The pipe-quadrupole, an alternative for high gradient interaction region quadrupole designs
}

\author{
J.M. van Oort and R.M. Scanlan \\ E.O. Lawrence Berkeley National Laboratory, 1 Cyclotron Road, Berkeley, CA 94720 -
}

\begin{abstract}
In the design of interaction region (IR) quadrupoles for high luminosity colliders such as the LHC or a possible upgrade of the Tevatron, the radiation heating of the coil windings is an important issue. Two obvious solutions to this problem can be chosen, the first is to reduce the heat load by added shielding, increased cooling with fins or using $\mathrm{Nb}_{3} \mathrm{Sn}$ to increase the temperature margin. The second solution eliminates the conductor from the areas with the highest radiation intensity, which are located on the symmetry-axes of the midplanes of the coils. A novel quadrupole design is presented, in which the conductor is wound on four half-moon shaped supports, forming elongated toroid sections. The assembly of the four shapes yields a quadrupole field with an active flux return path, and a void in the high radiation area. This void can be occupied by a liquid helium cooling pipe to lower the temperature of the windings from the inside. The coil layout, harmonic optimization and mechanical design are shown, together with the calculated temperature rise for the radiation load of the $\mathrm{LHC}$ interaction region quadrupoles.
\end{abstract}

\section{DESIGN PHILOSOPHY}

In interaction region quadrupoles, the highest intensity of the radiation is located on the primary symmetry-axes of the quadrupole field [1], depositing energy directly into the coils in the case of a normal cosine- $2 \theta$ coil layout, as illustrated in Fig. 1. A novel quadrupole design is presented here, based on the pipe-dipole design [2], in which the superconducting cable is wound on four halfmoon shaped supports, essentially forming elongated toroid sections. The assembly of the four shapes yields a quadrupole field with an active flux return path, and a void in the high radiation area. This void can be occupied by a liquid helium cooling pipe mounted on a radiation blocking heat sink to lower the temperature of the windings from the inside. The overall layout of such a magnet design with an effective bore diameter of $3 \mathrm{~cm}$ is shown in Fig. 2. The winding form can be made from either iron to enhance the field gradient, or from another non-magnetic metal for structural support only. The complex shape of the winding form is made by wire-cutting the cable slots out of a laminated block of material to avoid the heating due to eddy-currents.

\section{Manuscript received August 27, 1996.}

This work was supported by the Director, Office of Energy Research, Office of High Energy and Nuclear Physics, High Energy Physics Division, Department of Energy, under Contract No. DE-AC0376 SF00098.

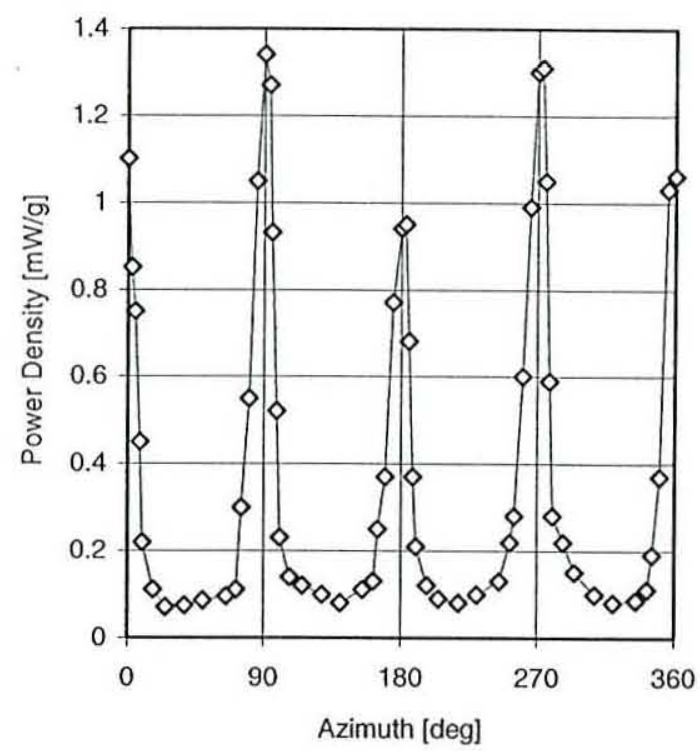

Fig. 1. Computed radiation load in the LHC interaction region quadrupoles [1]. The highest intensity of the radiation is in the middle of the coil blocks of the conventional cosine- $2 \theta$ quadrupole layout. The heat load from the radiation is computed from the beam induced energy deposition for the inner triplet magnets, shown is the power density for the innermost area of the conductor.

\section{CONDUCTOR LAYOUT AND STACKING}

When the coil design is compared to a cosine- $2 \theta$ quadrupole winding, the conductor stacking is done in reverse, the layers closest to the winding shape are wound on the mandrel first, stacking is done towards the beampipe.

The total assembly uses at least twice the amount of superconductor compared to a cosine-theta layout, but the overall cold-mass is smaller, and less susceptible to radiation heating, thus making this an ideal candidate for IR-quadrupoles in the detector region.

The coil shape is wound readily with small cables or tapes; also the packing factor will be higher than when large cables are used. All conductor layouts shown in this paper are based on a small 8 strand rectangular NbTi cable with a width of $2.72 \mathrm{~mm}$ and a thickness of $1.07 \mathrm{~mm}$. The cable is made of the standard SSC outer strand material and is wrapped with a $50 \%$ overlap polyamide tape with a thickness of $50 \mu \mathrm{m}$ for electrical insulation. 


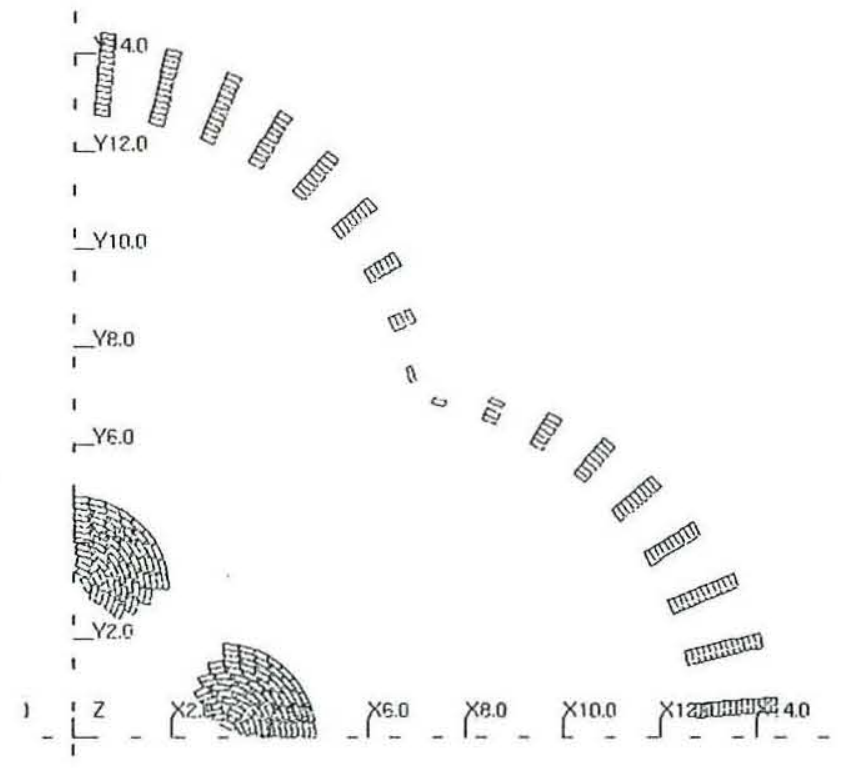

Fig 2. A solution for the conductor layout for a pipe-quadrupole (units are in $\mathrm{cm}$ ). The cable current is $3061 \mathrm{~A}$ and the field gradient is $250 \mathrm{~T} / \mathrm{m}$. The highest field reached is $8.23 \mathrm{~T}$ in the inner coil segment nearest the 45 degree symmetry line.

The coil optimization and field calculations have been done analytically, and were verified using the ANSYS [3] and Opera-3D [4] codes. The coil designs are all done with an overall $j_{C}$ for $\mathrm{NbTi}$ of $1000 \mathrm{~A} / \mathrm{mm} 2$ at $8 \mathrm{~T}$. This is a fairly high critical current value, but commercially available if the $\mathrm{Cu} / \mathrm{SC}$ ratio is kept around $0.3: 1$. Since the cables are primarily stacked in parallel to the magnetic field this coil shape can be wound using tape conductor without generating large losses.

\section{COIL END DESIGN}

The coil end design is similar to that of a conventional electrical motor winding. A metal fillet piece is placed against the end of the slotted winding form to provide support for the first layer wrapped around it. The fillet has two protruding edges to guide the outermost cables around the curved form. All subsequent layers are wound on top of the previous ones, and fixed in place with glass-fiber reinforced epoxy.

To demonstrate the winding process a model was made of one half quadrant using high density architectural foam. The winding slots were cut into the outer radius of a $24 \mathrm{~cm}$ long foam quarter bar, and the inner stacking radius was cut out. Next, the fillets were mounted onto the ends. The resulting winding form was used to demonstrate the winding sequence with the actual $\mathrm{NbTi}$ cable. No significant winding problems were encountered.

The accuracy of the cable positions is guaranteed by the precisely cut slots in the winding form for the outer radius. On the inner radius the precision is guaranteed by fitting the available space for each layer to the required width of the cables plus insulation, ensuring a snug fit for the cables.

\section{HARMONIC OPTIMIZATION}

The harmonic optimization is done by tapering the consecutive layers further inward along each stacking circle for increasing layers counting from the cooling pipe. This essentially offsets the start of each layer a certain distance with respect to the 45 degree symmetry line. This method provides an n-dimensional degree of freedom for the harmonic optimization, with $\mathrm{n}$ the amount of layers in the coil winding.

Further optimization can be achieved by varying the spacing between the cables in both the inner coil and outer coil, this was not used in the current design. All harmonic components of the field have been minimized below 1 unit at $1 \mathrm{~cm}$. Further optimization to minimize the unwanted components further (i.e. smaller than $1 \times 10^{-7}$ times the quadrupole component) remains to be done. This will be done by changing the slot spacing in the outer return coil, since the precision of these slots can be guaranteed due to the wire-cutting process of the part.

\section{MECHANICAL DESIGN AND PROTECTION ISSUES}

Since the forces primarily react radially, the mechanical design can be kept fairly simple. The whole coil structure can be surrounded by a thin iron yoke to prevent the small amount of flux from leaking out of the active return coil. The eight coil forms are manufactured by a wire-cutting (EDM) process, and mounted together after the coil winding is completed. Based on the required field gradient, the coil form can be manufactured out of iron or stainless steel. The thermal contraction of the coil form has to be kept close to that of the conductor, preferably slightly lower, ensuring that the conductor is held in place when the magnet is cooled to $4.2 \mathrm{~K}$.

An ANSYS finite element model was built of a coil quadrant to check the Lorentz loads and stresses during cooldown and operation. The maximum stress on the conductor is in the order of $85 \mathrm{MPa}$ for a field gradient of $250 \mathrm{~T} / \mathrm{m}$. The coil end load will be supported by an end piece that is mounted directly onto the coil winding form. This allows a separate end shoe to support the windings in the end region without having to support the entire axial load on the superconductor.

The compressive end load on the conductor section is preset with tensioned bolts mounted to the side of the outermost coil forms. The coils can be wound as either four quadrants or eight half-quadrants. Using eight halfquadrants makes the stacking process somewhat easier, but will require doubling the number of joints in the magnet. A benefit of using eight components is that the protection diodes can be placed across each half-quadrant, lowering the voltage the coil will see during a quench. For the design shown in Fig. 2. with a cable current of $3061 \mathrm{~A}$, a total of 
9.3 kA-turns per quadrant is needed for a gradient of $250 \mathrm{~T} / \mathrm{m}$.

\section{COIL HEATING DUE TO THE RADIATION LOAD}

To illustrate the desirability of an alternate quadrupole design more resistant to coil heating, a thermal analysis was performed of the effect of the radiation heat load on the coils of a generic cosine- $2 \theta$ design quadrupole for the LHC. The radiation load was taken from calculations from Mokhov et.al [1], and compared to previous models on Hadron showers by Burnod et.al. [5],[6]. The average overall heat load is in the order of $10 \mathrm{~mW} / \mathrm{cm}^{3}$, with a distribution profile as shown in Fig.1. The heat load distribution was computed as an average per cable and applied to a non-linear thermal FEM model at $1.9 \mathrm{~K}$.

The resulting temperature rise in the coils was computed at about $0.7 \mathrm{~K}$ (model) to $2.29 \mathrm{~K} \mathrm{[7]} \mathrm{in} \mathrm{the} \mathrm{inner}$ layer, effectively raising the coil temperature above the critical temperature of the superfluid. The same procedure applied to the pipe quadrupole design yields a temperature increase of the order of $3 \mathrm{mK}$. This illustrates the effectiveness of the cooling pipe and the removal of conductor from the most heavily irradiated areas. Next, the radiation load profile was modified to include a tungsten beam block; this would further reduce the amount of heat deposited in the coil by a factor of 2-3 by blocking the radiation before it reaches the conductor. This requires that the beam block itself is well cooled and not in direct thermal contact with the coil segments. In the pipe magnet this is achieved by cooling the tungsten directly with a forced helium flow on the back side of the beam block.

\section{COMPARISON WITH CONVENTIONAL QUADRUPOLE DESIGNS}

When compared with a conventional cosine- $2 \theta$ design or block quadrupole, the pipe quadrupole is fairly inefficient with regard to the conductor volume. This is primarily due to the active flux return coil on the outer radius, which doubles the amount of conductor required. Furthermore, moving conductor away from the beam pipe to allow for space for the cooling structure also reduces the overall efficiency of the design.

Compared to an optimized high gradient quadrupole design [8] with the same bore diameter, with a cable current of $6043 \mathrm{~A}$, and a total of 54 turns, the pipe design uses 2.8 times the conductor volume, with a resulting transfer function of $12.24 \mathrm{~T} / \mathrm{m}-\mathrm{kA}$, where the cosine- $2 \theta$ design has a transfer function of $41.37 \mathrm{~T} / \mathrm{m}-\mathrm{kA}$.

However, the outer radius of the coil of the cosine- $2 \theta$ design is at $11.7 \mathrm{~cm}$, with an OD of the iron yoke of $30 \mathrm{~cm}$. The OD of the pipe design is $28.6 \mathrm{~cm}$, with no need for a circular outer shape. This allows the total cold mass to be smaller in size, which is beneficial for a magnet in the detector region. The pipe quadrupole can be made smaller in overall size by reducing the outer layer stacking radius at the expense of a small amount of field gradient. If a conductor is used with sufficient margin, this would allow the magnet design to become even more compact as compared to a cosine- $2 \theta$ design.

\section{CONCLUSIONS}

A high gradient quadrupole with a "pipe" layout should be considered as a promising candidate for future large collider interaction regions. The design can be optimized to obtain the required field-quality and the conductor is cooled in the region where the radiation heat load is the largest.

The temperature rise in the coil windings is reduced dramatically by the insertion of a tungsten beam block and a liquid helium cooling pipe. The computed maximum temperature in the coil dropped from $0.7 \mathrm{~K}$ for a cosine- $2 \theta$ design to a $3 \mathrm{mK}$ for the pipe design.

The overall structure is slightly smaller than a conventional $\operatorname{cosine}-2 \theta$ design quadrupole with a comparable field gradient, and can be reduced even further in size by decreasing the outer coil segment stacking radius at the expense of a small amount of field gradient.

The pipe design is less efficient than the cosine- $2 \theta$ design; for a similar field gradient 2.8 times more conductor is needed. This is due to the less efficient transfer function of the inner coil segment, and the double amount of conductor needed for the outer return coil.

The windability of the design and the end regions has been demonstrated with a small dummy model. No significant problems were encountered during the coil winding. Also the winding of the cable around the end regions proved to be possible without problems.

Future plans at LBNL include a small, one-quadrant demonstration coil with NbTi superconductor on a wire-cut metal winding form

\section{REFERENCES}

[1] N.Mokhov, "Beam induced energy deposition in the LHC inner triplet magnets", IR Quad Design Workshop, Fermi National Accelerator Laboratory, Batavia, Illinois, 1995.

[2] E. Badea, P.M. McIntyre and S. Pissanetzky, "The Pipe Magnet Compact 13 Tesla Dual Dipole for Future Hadron Colliders", presented at the Annual High Field Accelerator Magnet Workshop, Berkeley CA, March 9-11, 1993.

[3] ANSYS $®$ Swanson Analysis Systems Inc.

[4] Opera-3D @Vector Fields Ltd.

[5] L.Burnod, "Problems arising from beam losses in superconducting colliders", Internal note EMA89/13, CERN, Geneva, Switzerland, 1989.

[6] L.Burnod and J.B. Jeanneret, "Beam losses in the SPS and the LHC due to beam-gas and beam-beam collisions", LHC-note 91, CERN, Geneva, Switzerland, 1989.

[7] R. Bossert et al., "Development of a high gradient quadrupole for the LHC interaction regions", this conference.

[8] A.D. McInturff, J.M. van Oort and R.M. Scanlan, "Two alternate high gradient quadrupoles; and upgraded Tevatron IR and a pipe design", IEEE Particle Accelerator Conference, Dallas TX, p. 1402, 1995. 